\title{
Pharmacological Inhibition of mTORC1 Suppresses Anatomical, Cellular, and Behavioral Abnormalities in Neural-Specific Pten Knock-Out Mice
}

\author{
Jing Zhou, ${ }^{1}$ Jacqueline Blundell, ${ }^{2}$ Shiori Ogawa, ${ }^{3}$ Chang-Hyuk Kwon, ${ }^{1}$ Wei Zhang, ${ }^{1}$ Christopher Sinton, ${ }^{3}$ \\ Craig M. Powell, ${ }^{2,4}$ and Luis F. Parada ${ }^{1}$ \\ ${ }^{1}$ Department of Developmental Biology and Kent Waldrep Foundation Center for Basic Neuroscience Research on Nerve Growth and Regeneration, and \\ Departments of ${ }^{2}$ Neurology, ${ }^{3}$ Internal Medicine, and ${ }^{4}$ Psychiatry, University of Texas Southwestern Medical Center, Dallas, Texas 75390
}

\begin{abstract}
PTEN (phosphatase and tensin homolog deleted on chromosome ten) is a lipid phosphatase that counteracts the function of phosphatidylinositol-3 kinase (PI3K). Loss of function of PTEN results in constitutive activation of AKT and downstream effectors and correlates with many human cancers, as well as various brain disorders, including macrocephaly, seizures, Lhermitte-Duclos disease, and autism. We previously generated a conditional Pten knock-out mouse line with Pten loss in limited postmitotic neurons in the cortex and hippocampus. Pten-null neurons developed neuronal hypertrophy and loss of neuronal polarity. The mutant mice exhibited macrocephaly and behavioral abnormalities reminiscent of certain features of human autism. Here, we report that rapamycin, a specific inhibitor of mammalian target of rapamycin complex 1 (mTORC1), can prevent and reverse neuronal hypertrophy, resulting in the amelioration of a subset of PTEN-associated abnormal behaviors, providing evidence that the mTORC1 pathway downstream of PTEN is critical for this complex phenotype.
\end{abstract}

Key words: PTEN; tuberous sclerosis complex; autism; macrocephaly; neuronal hypertrophy; neuronal polarity

\section{Introduction}

PTEN (phosphatase and tensin homolog deleted on chromosome ten) is a major negative regulator of the phosphatidylinositol-3 kinase (PI3K)/AKT pathway. Its primary function is to counteract the kinase function of PI3K and therefore inhibit the activation of AKT. Individuals bearing germ-line PTEN mutations are prone to many human cancers (Ali et al., 1999), as well as mental disorders, including macrocephaly, seizures, mental retardation, and Lhermitte-Duclos disease (Marsh et al., 1999; Zhou et al., 2003). Recently, PTEN mutations have also been linked to cases of autism, particularly in a subset of patients with macrocephaly, suggesting that mutations in this gene might be one of the genetic risk factors for human autism spectrum disorders (ASDs) (Goffin et al., 2001; Butler et al., 2005; Herman et al., 2007a,b).

We reported previously a strain of conditional Nse-cre; Pten $^{\text {loxP/loxP }}$ knock-out mice (Pten mutant mice hereafter) with Pten ablation in a subset of postmitotic neurons in the cortex and hippocampus. Pten mutant mice develop macroceph-

Received Nov. 28, 2008; accepted Dec. 23, 2008.

This work was supported by the Simons Foundation (L.F.P.) and Autism Speaks (C.M.P.). We thank Dr. Tak Mak for the Pten ${ }^{\text {IoxP }}$ mice, Dr. David Kwiatkowski for the $T s{ }^{\text {IoxP }}$ mice, Dr. Suzanne J. Baker for sharing the Nse-cre mice, Dr. Renee McKay for helpful advice, and Yanjiao Li, Shawna Kennedy, and Steve McKinnon for technical assistance. We thank Sharon Matheny for helpful discussions.

Correspondence should be addressed to Luis F. Parada, Department of Developmental Biology and Kent Waldrep Foundation Center for Basic Neuroscience Research on Nerve Growth and Regeneration, University of Texas Southwestern Medical Center, Dallas, TX75390.E-mail: luis.parada@utsouthwestern.edu.

DOI:10.1523/JNEUROSCI.5685-08.2009

Copyright $\odot 2009$ Society for Neuroscience $\quad$ 0270-6474/09/291773-11\$15.00/0 aly and additional behavioral abnormalities reminiscent of human autism, including reduced social activity, increased anxiety, and sporadic seizures (Kwon et al., 2006a; Ogawa et al., 2007). At the cellular level, we observed two major morphological changes after in vivo loss of Pten: loss of neuronal polarity and neuronal hypertrophy.

One important question is which of the downstream pathways of PI3K/AKT mediates these cellular and behavioral changes. Among the various PI3K/AKT downstream pathways, tuberous sclerosis complex $1 / 2$ (TSC1/2) is an attractive candidate. It is known that TSC1 and TSC2 function together as a dimer. AKT directly phosphorylates TSC2 and releases the suppression of TSC1/TSC2 on mammalian target of rapamycin complex 1 (mTORC1) (Inoki et al., 2002; Manning et al., 2002; Potter et al., 2002). We were particularly interested in this AKT downstream pathway because of two existing facts. First, mutation of either TSC1 or TSC2 gene causes tuberous sclerosis in humans, in which 25-50\% of patients show autistic syndrome (Smalley et al., 1992; Smalley, 1998; Wiznitzer, 2004). Second, it has been shown that loss of Tsc1 results in neuronal hypertrophy both in vitro and in vivo (Jaworski et al., 2005; Tavazoie et al., 2005; Meikle et al., 2007). In addition, a recent study suggests that the TSC/ mTORC1 pathway is involved in controlling neuronal polarity (Choi et al., 2008).

In the current study, we examined the role of the TSC/ mTORC1 pathway in Pten mutant mice through the use of a specific mTORC1 inhibitor, rapamycin. Our results indicate a major contribution of this pathway to the abnormal cellular mor- 
phology. Pharmacologic attenuation of the pathway in Pten mutants by rapamycin has surprising consequences on neuronal hypertrophy, seizures, and social behavior. In addition, we show that ablation of Tsc1 using the identical Nse-cre transgenic mouse line mimics the morphological features observed with the Pten mutant mice. These data point to a common signal transduction pathway that is potentially responsible for the autism-like symptoms in individuals bearing TSC1/2 or PTEN mutations and suggest a possible treatment strategy for this subset of autism patients.

\section{Materials and Methods}

Mice and histology. Pten ${ }^{\operatorname{lox} P}$ mice (Suzuki et al., 2001) were a gift from Dr. Tak Mak (University of Toronto, Toronto, Ontario, Canada), and the Nse-cre line was generated in the laboratory of Dr. Suzanne J. Baker (St. Jude Children's Research Hospital, Memphis, TN) and characterized in our laboratory (Kwon et al., 2006b). Both lines have been maintained in $C 57 B L / 6$ inbred background. Pten mutant mice (Nsecre;Pten $\left.{ }^{\operatorname{lox} P / \operatorname{lox} P}\right)$ were generated by breeding male Pten ${ }^{\operatorname{lox} P / l o x P}$ mice and female Nse-cre; Pten $^{\text {loxP } /+}$ mice. Controls used here were littermates either without cre or in a few cases Nsecre;Pten ${ }^{\text {loxP } /+}$. Tscl ${ }^{\text {loxP }}$ mice were a gift from Dr. David Kwiatkowski (Harvard Medical School, Boston, MA) and were crossed with the Nsecre line by a similar strategy. For preparation of brain sections, mice were intracardially perfused with ice-cold PBS, followed by $4 \%$ paraformaldehyde (PFA). Brains were dissected out immediately after perfusion and kept in $4 \%$ PFA at $4^{\circ} \mathrm{C}$ for additional use. For paraffin sectioning, halfbrains were processed, embedded in paraffin, and sectioned sagittally at 5 $\mu \mathrm{m}$ thickness. For vibratome floating sectioning, brains were embedded in $3 \%$ agarose and then cut coronally at $50 \mu \mathrm{m}$ thickness. All mouse protocols were approved by the Institutional Animal Care and Research Advisory Committee at the University of Texas Southwestern Medical Center.

Rapamycin injection. Rapamycin powder (Sirolimus; LC Laboratories) was dissolved in ethanol and stored at a stock concentration of $25 \mathrm{mg} / \mathrm{ml}$ in aliquots at $-20^{\circ} \mathrm{C}$. Working solution was prepared freshly before use with a final concentration of $1 \mathrm{mg} / \mathrm{ml}$ rapamycin in $4 \%$ ethanol, $5 \%$ Tween 80, and 5\% PEG400 (Kwon et al., 2003). Mice were injected intraperitoneally with either rapamycin $(10 \mathrm{mg} / \mathrm{kg}$ body weight $)$ or vehicle once per day for 5 consecutive days per week (Monday to Friday).

Western blotting. Hippocampus and cortex were isolated separately from mouse brain and snap frozen in liquid nitrogen. To prepare the lysate, frozen tissues were homogenized in lysis buffer containing $20 \mathrm{~mm}$ Tris-HCl, 150 mm NaCl, 1 mm EDTA, 1 mm EGTA, 1\% Triton X-100, proteinase inhibitor (Roche), Ser/Thr phosphatase inhibitor, and Tyr phosphatase inhibitor (Millipore). Homogenate was centrifuged at 14,000 rpm for $30 \mathrm{~min}$ and supernatant was collected. Protein concentration was determined by using BCA kit (Pierce). For Western blotting, 20 or $50 \mu \mathrm{g}$ of sample was loaded onto SDS-PAGE gel. Antibodies used for Western blotting were against P-AKT-Ser473, AKT, P-S6 (Ser235/ 236), or S6 (Cell Signaling Technology), or $\beta$-actin (Sigma). Signal was developed by ChemiGlow West reagent (Alpha Innotech) and visualized by Eastman Kodak Image Station 2000R.

Immunohistochemistry. Triplicate sections with comparable anatomy were chosen from controls and mutants. Antibodies used for immunohistochemistry (IHC) were against PTEN, P-AKT, P-S6 (Cell Signaling Technology), Calbindin (Swant), Synapsin I (Millipore Bioscience Research Reagents), or SMI 311 (Covance Research Products). For paraffin sections, we used microwave antigen retrieval for all antibodies. We visualized the primary antibodies by treating the sections with biotinylated secondary antibody, followed by amplification with peroxidaseconjugated avidin and DAB substrate (Vector Laboratories). DABstained sections were counterstained with hematoxylin. For vibratome sections, we detected the primary antibodies by secondary antibodies conjugated with cyanine 3 or cyanine 2 (Jackson ImmunoResearch).

Measurements. All the measurements were performed using the MetaMorph software (Universal Imaging). We measured nuclear diameter of dentate granule neurons as an indication of soma size (Kwon et al., 2003). To estimate the thickness of the mossy fiber tract, we measured the width of out-coming axonal bundles from the dentate gyrus that were double labeled with Synapsin I and Calbindin. To assess dendritic size, we measured the length of Calbindin-stained neuronal processes into the molecular layer (ML). For cortical neurons, we measured SMI 311-positive neurons in wild-type controls and compared them with SMI 311-positive and PTEN-negative neurons in Pten mutants. For each animal, only neurons in somatosensory cortex were analyzed. All data were analyzed with ANOVA, followed by post hoc Student's $t$ test.

Golgi staining. We performed Golgi staining, image analysis, and dendritic caliber and spine density quantification as described previously (Luikart et al., 2005).

Timm's staining. For Timm's staining, we intracardially perfused the mice with ice-cold $0.37 \%(\mathrm{w} / \mathrm{v})$ sodium sulfide, followed by $4 \%$ PFA. We dissected out the brain, postfixed in 4\% PFA overnight, and cryoprotected in $30 \%(\mathrm{w} / \mathrm{v})$ sucrose in PBS for $2-3 \mathrm{~d}$. We made $16-\mu \mathrm{m}$-thick coronal cryostat sections and performed a modified Timm's staining as described previously (Danscher et al., 2004).

Behavioral tests. Mutant mice were studied along with littermate controls in four groups: vehicle-treated controls, vehicle-treated Pten mutant mice, rapamycin-treated controls, and rapamycin-treated Pten mutant mice. Behavioral tests were performed as described previously (Kwon et al., 2006a). The open-field test was performed for $10 \mathrm{~min}$ in a brightly lit, $48 \times 48 \mathrm{~cm}^{2}$ white plastic arena with a center zone defined as a $15 \times 15 \mathrm{~cm}^{2}$ square. Time spent in the center zone and total travel distance were recorded using video-tracking software (Noldus). The social interaction tests were performed in a neutral cage in the dark under 
A

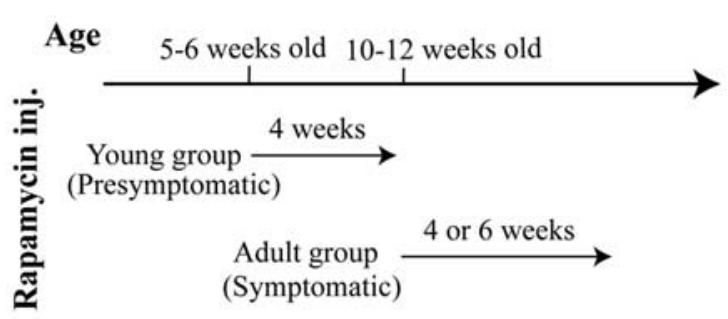

B
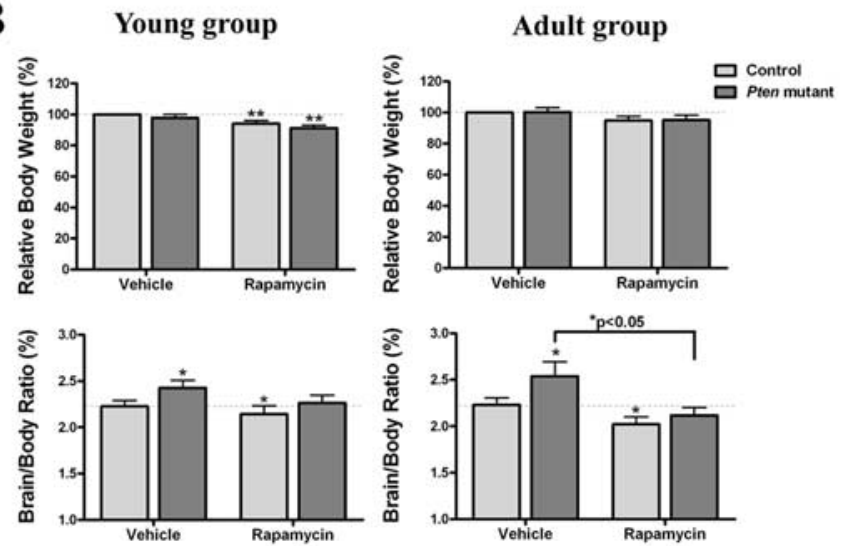

C
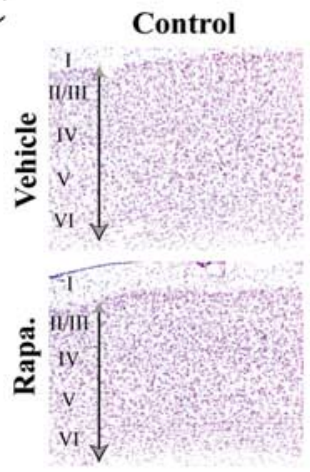

Control

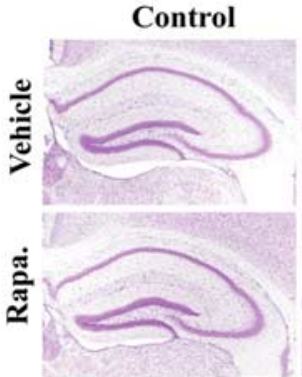

Pten mutant

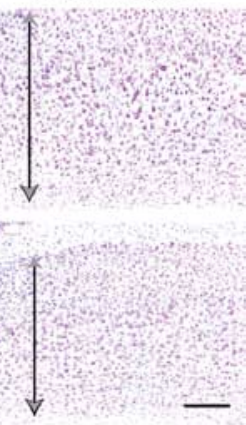

Pten mutant

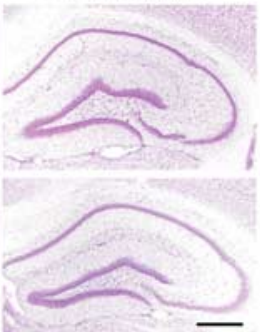

Figure 2. Rapamycin can prevent or reverse macrocephaly in Pten mutant mice. A, Rapamycin injection strategy. Rapamycin was applied to two cohorts of mice at different ages: a young group (5- 6 weeks old) and an adult group (10-12 weeks old). Mutants and controls injected with either vehicle or $10 \mathrm{mg} / \mathrm{kg}$ rapamycin were analyzed after 4 or 6 weeks of injections. B, Rapamycin prevents or reverses the brain enlargement of Pten mutant mice. The effect of rapamycin on brain and body weight was measured after 4 weeks for the young group or after 6 weeks for the adult group ( $n=15$ mice per group for body weight measurement, $n=8$ mice per group for brain/body ratio). Data are mean \pm SEM and were analyzed by paired $t$ test. ${ }^{*} p<0.05$ and ${ }^{* *} p<0.01$ compared with control mice treated with vehicle or between groups as indicated. C, Representative Niss staining shows that rapamycin (Rapa) treatment suppressed the enlargement of the cortex (scale bar, $200 \mu \mathrm{m}$ ) and hippocampus (scale bar, $500 \mu \mathrm{m}$ ) when applied to young Pten mutant mice for 4 weeks. Arrows show thickness of cortical layers $\mathrm{II}-\mathrm{Vl}$.

red light by pairing a test mouse with a male juvenile mouse. During a 2 min time window, the time that the testing mouse actively approached and sniffed the social target was recorded. After this initial interaction, the test was repeated after $3 \mathrm{~d}$ by reintroducing the same social target. The above behavioral tests were performed blindly to genotype and treatment.

Electroencephalogram/electromyogram recording. For electroencephalogram/electromyogram (EEG/EMG) analysis, Pten mutant $(n=12)$ and wild-type $(n=3)$ mice were anesthetized and surgically implanted for long-term EEG/EMG monitoring as described previously (Chemelli et al., 1999). The design for the EEG/EMG implant allowed precise insertion of electrodes, targeting the frontal and occipital cortices at a consistent depth, just touching the dura, while minimizing surgical trauma. Mice were housed individually under a $12 \mathrm{~h}$ light/dark cycle at $25^{\circ} \mathrm{C}$ [lights on, circadian time (CT) 0000], with food and water being replenished as necessary at CT 1200 each day, but they were not otherwise disturbed. Mice were habituated to the recording conditions for 2 weeks before baseline EEG/EMG signals were recorded over a period of $3 \mathrm{~d}$, beginning at lights off (CT 1200). In the following week, the mice were divided into two groups: a rapamycin treatment group $(n=6$ mutants, $n=3$ controls) and a vehicle control group ( $n=6$ mutants). Each week, the animals were intraperitoneally injected with either rapamycin or vehicle once daily between 10:00 and 11:00 A.M. for 5 successive days. The EEG/EMG was recorded from the second day of the injection continuing for $3 \mathrm{~d}$, and this procedure was repeated for 4 weeks to evaluate the chronic effect of the drug. No adverse effects from the injection were observed in either group, and the body weights remained stable throughout the study. Subsequently, the EEG/EMG record was visually screened for seizure epochs. Seizures were characterized as a spike-wave pattern on the EEG, typically accompanied by atonic periods or sustained rhythmic contractions on the EMG. Each seizure lasting for $2 \mathrm{~s}$ or more was noted. The total number of seizures during the $12 \mathrm{~h}$ dark and light periods was analyzed, as well as the time of occurrence and the duration of each seizure. One-way ANOVA with Bonferroni's multiple comparison as a post hoc test was applied to analyze differences within groups.

\section{Results}

Rapamycin effectively blocks mTORC1 signaling in the CNS

A dose-response experiment was performed to determine an effective rapamycin concentration that would inhibit mTORC1 activity in mouse cortex and hippocampus. Rapamycin (Sirolimus) (Heitman et al., 1991) was administered by intraperitoneal injection for 5 consecutive days at various concentrations. Mice were killed on the last day of injection for analysis. As readout for mTORC1 activity, we assayed levels of phospho-Ser235/236-S6 (P-S6 hereafter) and determined that $10 \mathrm{mg} / \mathrm{kg}$ rapamycin effectively reduced mTORC1 signaling in both hippocampus and cortex (Fig. $1 \mathrm{~A}$ ). In addition, $10 \mathrm{mg} / \mathrm{kg}$ rapamycin was also sufficient to attenuate mTORC1 signaling in Pten mutant brains, in which mTORC1 was highly activated (Fig. $1 B, C$ ). We therefore adopted this rapamycin dosage in all subsequent studies.

\section{Rapamycin can prevent or reverse macrocephaly in Pten mutant mice}

In our mouse model, deletion of Pten is mainly restricted to subsets of postmitotic neurons in cortex and hippocampus (Kwon et al., 2006b). By using a Rosa26-stop-lacZ reporter mouse line (Soriano, 1999), we found that cre activity in the brain mainly developed postnatally (supplemental Fig. 1, available at www. jneurosci.org as supplemental material). Only minimal cre activity could be detected at early postnatal days but increased dramatically during the second and third postnatal weeks, reaching maximal activity at 4 weeks.

In our previous study, Pten mutant mice were carefully monitored and shown to progressively develop macrocephaly that is detectable as early as $4-5$ weeks of age, reaching statistical significance at 2 months of age (Kwon et al., 2006a). This timing correlates with the appearance of abnormal behavior, which appears 
at 5-6 weeks of age and is fully penetrant by 2-3 months of age. We chose to apply rapamycin to two cohorts of mutant and control mice: a presymptomatic young group (5-6 weeks old) and a symptomatic adult group (10-12 weeks old). Thus, in the former group, rapamycin activity would be present at the earliest manifestation of abnormality and would be tested for prevention of symptoms. In the latter group, rapamycin delivery commenced when symptoms were fully penetrant to test the potential for reversal. For each group, mutants and age-matched controls were injected with either vehicle or 10 $\mathrm{mg} / \mathrm{kg}$ rapamycin. Mice were killed after either 4 or 6 weeks of drug treatment for analysis as illustrated in Figure $2 \mathrm{~A}$.

Overall, the mice appeared healthy during rapamycin administration, although slight growth retardation was observed after long-term treatment. In the young group, rapamycin-injected mice showed a 5-9\% reduction in body weight compared with vehicle-injected controls. This effect became less obvious in the adult group, which shared a similar trend but did not reach statistical significance. To account for the overall effect of rapamycin on growth, brain/body weight ratio was used to assess relative brain growth (Fig. $2 B)$. In the young group, the vehicletreated mutant mice already showed significantly increased brain/body weight ratio compared with control mice by the fourth week of injection. In contrast, the mutants receiving rapamycin showed normal brain/body weight ratio. In the adult group, the same effect was observed, although the macrocephaly (17\% increase in brain weight) was more dramatic than in the young group (7\% increase in brain weight) (Fig. 2B). As described previously, the enlarged brain size is mainly the consequence of Pten null cellautonomous overgrowth in the two brain regions in which cre activity is confined (cortex and hippocampus). Consistent with the brain weight data, the enlargement of cortex and hippocampus in mutants is controlled by rapamycin, as shown by Nissl staining of the treated young group of mice (Fig. 2C).

Rapamycin suppresses hippocampal granule cell hypertrophy Using various techniques, we have assessed the changes in cell morphology caused by Pten loss and demonstrated that brain enlargement is not only attributable to enlarged soma size but also neuronal process hypertrophy, including increased dendritic size and thickened axon bundles (Kwon et al., 2006a). We therefore first examined the effect of rapamycin on neuronal hypertrophy in the presymptomatic group of mice.

On tissue sections, the cellular boundary of granule cells in the dentate gyrus is not clearly identifiable because the nuclei of granule neurons are prominent and surrounded by a thin layer of cytosol. We therefore measured nuclear diameter to reflect soma size (Kwon et al., 2003, 2006a). PTEN immunostaining illustrates that Pten-negative cells (blue cells, hematoxylin counterstained) are obviously larger than Pten-positive cells (brown cells), whereas the soma hypertrophy does not develop in mutant mice undergoing rapamycin treatment (Fig. $3 A, B$ ).

To examine the effect of the drug on neuronal processes, we again took advantage of the well established circuitry in the hippocampus. The granule cells of the dentate gyrus spread their dendrites out toward the molecular layer, whereas the axons form the mossy fiber tract that projects through the polymorphic layer to synapse onto the CA3 region. The mossy fiber tract was visualized by IHC using antibodies to Synapsin I, a presynaptic marker, and to Calbindin, which is expressed in both soma and processes. The increased thickness of the mossy fiber tract seen in vehicle-treated mutants was dramatically suppressed by rapamycin administration (Fig. 4A,B). This result was confirmed by Timm's staining, which specifically highlights the mossy fiber tract (Fig. 4C). Dendritic growth was measured by the thickness of the ML, defined here as the length of Calbindin-stained neuronal processes into the ML. The data indicate that dendritic overgrowth was also reduced by rapamycin (Fig. 4A,D).

We further observed that, at the end of treatment, four of eight mutants treated with vehicle exhibited ectopic Synapsin I reactivity in the inner ML (Fig. 4A, arrowheads), reflecting loss of polarity in granule neurons. This is a common feature of older mutant mice (Kwon et al., 2006a). Interestingly, none of the six rapamycin-treated mutants showed ectopic Synapsin I. Similar results were obtained with Timm's staining, with two of five mutants treated with vehicle showing ectopic staining in the inner ML, whereas none of the four mutants treated with rapamycin showed this ectopic staining. Together, these data indicate that, when administered to presymptomatic mice, rapamycin can sup- 

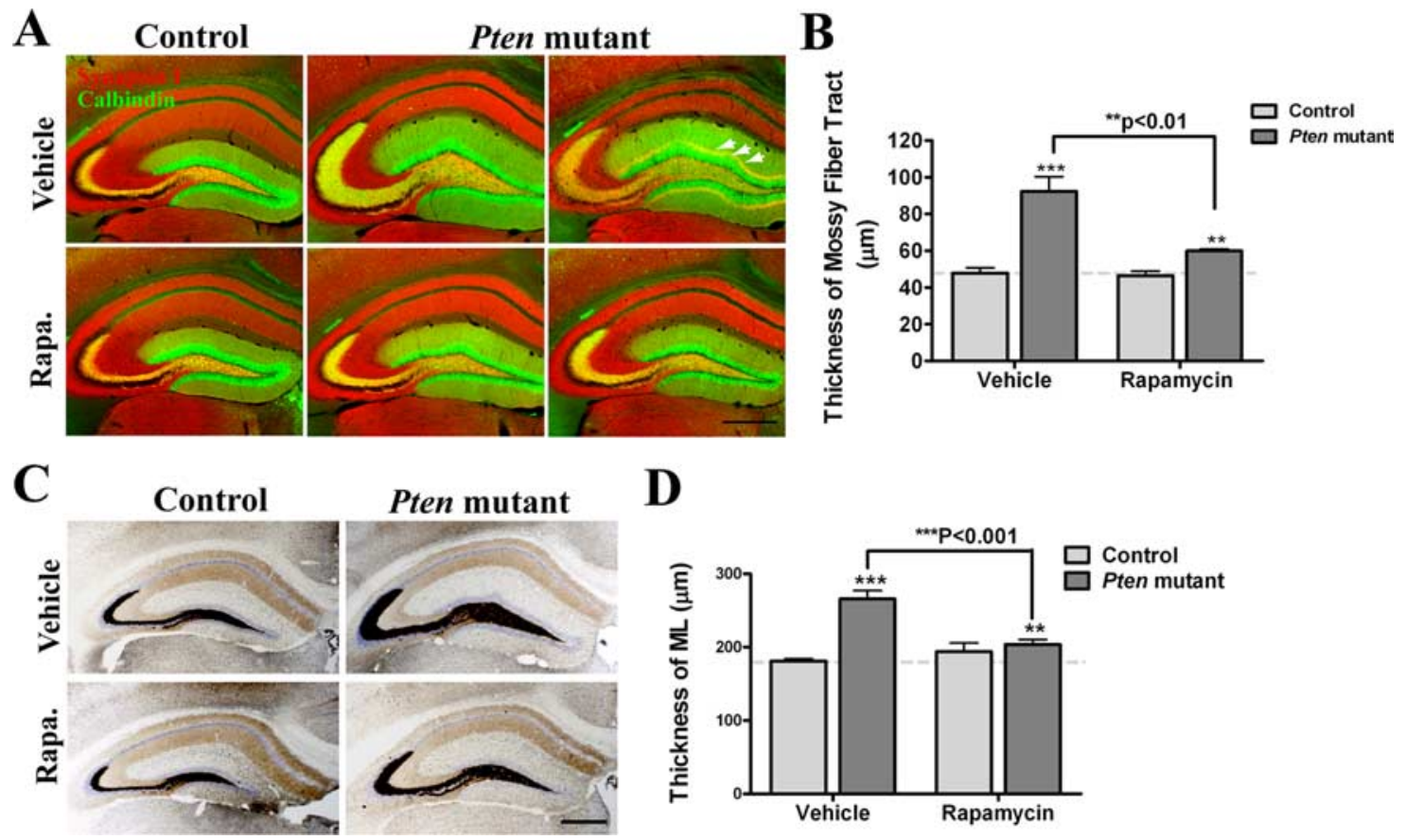

Figure 4. Rapamycin inhibits dendritic and axonal hypertrophy of dentate granule cells in young Pten mutant mice. A, Immunostaining on 50- $\mu \mathrm{m}$-floating sections for Synapsin I (red) and Calbindin (green) shows reduced dendritic and axonal hypertrophy in Pten mutants after rapamycin treatment. Ectopic Synapsin I staining (arrowhead) in the inner molecular layer was observed in some vehicle-treated Pten mutants. Scale bar, $500 \mu \mathrm{m}$. B, Quantification reveals that increased thickness of mossy fiber tract in Pten mutant mice was significantly reduced after rapamycin treatment. The thickness of mossy fiber tracts is defined by the width of the axon bundle coming out of the dentate gyrus in Synapsin I and Calbindin double-stained sections. $n=8$ mice for vehicle-treated groups; $n=6$ mice for rapamycin-treated groups. C, Timm's staining to visualize mossy fiber tract. $n=4$ or 5 mice were examined per group. Scale bar, $500 \mu \mathrm{m}$. $\boldsymbol{D}$, Increased thickness of the molecular layer in Pten mutant mice was significantly reduced after rapamycin treatment. Data are mean \pm SEM and were analyzed by ANOVA, followed by post hoc $t$ test. ${ }^{* *} p<$ 0.01 and $^{* * *} p<0.001$ compared with control mice treated with vehicle or between groups as indicated.

press the development of neuronal process hypertrophy and the loss of neuronal polarity caused by Pten ablation.

\section{Rapamycin inhibits cortical neuron hypertrophy}

Another region affected in the Pten mutant mice is the cortex, in which cre expression is restricted mainly to layers III-V with $\sim 30-60 \%$ neurons bearing cre activity. We examined pyramidal cells in somatosensory cortex of the presymptomatic group of mice. Neuronal morphology was visualized by IHC with antibodies against SMI 311 and PTEN. SMI 311 is a pan-neuronal neurofilament antibody that detects a subset of pyramidal cells in cortical layers III and V (Ulfig et al., 1998). In mutant mice, most Pten-null pyramidal cells, if not all, had obviously larger somata and were often accompanied by brighter staining of SMI 311 (Fig. $5 A$, arrowhead). Similar phenomena have been reported for neurons that lack $T s c 1$, suggesting neuronal hypertrophy with increased expression of neurofilament (Meikle et al., 2007). Compared with controls, the mutant samples showed overall increased SMI 311 staining, whereas with rapamycin treatment, the SMI 311 signal was reduced, as were soma size and thickness of the dendritic caliber (Fig. $5 B-D$, arrow). Therefore, rapamycin impedes development of abnormalities caused by Pten loss in cortical neurons as well.

\section{Rapamycin reverses dentate gyrus enlargement}

In later stages, we observed more dramatic neuronal abnormalities in the Pten mutants. By 4 months of age, mutants showed abnormal foliation of the hippocampal dentate granule layer and compression of the CA1 region (Kwon et al., 2006a). To evaluate whether rapamycin could reverse already established morphological abnormalities, we treated a second cohort of mice begin- ning at 10-12 weeks of age (adult group) for 4-6 weeks. Our results indicate that rapamycin was able to significantly reverse neuronal hypertrophy in older mice, resulting in restoration of much of the hippocampal structure (Fig. 6A). However, some compression of the CA1 region still remained. In contrast to the younger cohort study, ectopic Synapsin I staining in the ML persisted in the drug-treated cohort even after 6 weeks of rapamycin treatment (Fig. 6A, arrowheads). These data indicate the sustained loss of polarity of granular cells. Nonetheless, the extent of the Synapsin I staining in the ML was reduced and mainly restricted to the inner ML. The enlarged cell size of Pten-null granule cells was also progressively reversed by rapamycin treatment (Fig. 6B) (supplemental Fig. 2, available at www.jneurosci.org as supplemental material). In addition, the granule cell layer partially regained its tight organization, with less extracellular space that was probably previously occupied by the enlarged dendritic processes. To further visualize individual cell morphology, we performed Golgi staining. Consistent with our previous data (Kwon et al., 2006a), granule cells in mutant mice are globally enlarged. After rapamycin treatment, enlarged dendritic size was reversed, accompanied by reduced dendritic caliber thickness and spine density (Fig. 6C,D). However, in both vehicle- and rapamycin-treated samples, granule cells with multiple processes into polymorphic layers were occasionally observed, suggesting loss of polarity (Fig. 6C, arrowhead). This, together with the Synapsin I staining result, suggests that rapamycin, when applied to older mice, cannot reverse loss of polarity. It is possible that, in later stages, once the neurons lose polarity it is not fully reversible in vivo. However, we cannot exclude the possibility that other AKT downstream effectors are also involved. 


\section{Beneficial effects of rapamycin} on behavior

We previously demonstrated that Pten mutant mice have increased anxiety and impaired social behavior in a series of experimental paradigms (Kwon et al., 2006a). We therefore tested whether rapamycin treatment in a young cohort could prevent development of these abnormal behaviors. Because, at the cellular level, the effects of rapamycin were gradual, we chose to examine behaviors during the 4 th week of drug administration.

Restricted by the narrow time window, we performed specific behavioral tests to examine the effects of rapamycin treatment mainly on anxiety and social interest in mice. The open-field test measures anxiety in mice. Consistent with our previous findings, vehicle-treated mutant mice spent most of the time in the corner or along the edge of the open field and significantly less time in the center compared with controls, indicating increased anxiety in the mutants (Fig. 7A). Interestingly, after treatment with rapamycin, this difference was no longer apparent: a clear trend of increased time spent in the center was observed in mutant mice treated with rapamycin versus mutants treated with vehicle. As a control for possible locomotor defects, we measured the distances traveled by the four cohorts of mice. We found that rapamycin-treated mice traveled comparable distances in the test as vehicletreated controls, suggesting normal locomotor activity (supplemental Fig. 3, available at www.jneurosci.org as supplemental material). Therefore, rapamycin treatment reduced anxiety of Pten mutant mice.

Mice were also examined in a test for reciprocal social interaction. As before, vehicle-treated mutants showed a signifi-

cant decrease in social interaction compared with controls. Rapamycin treatment produced a clear increase in social interaction in the mutants. This effect of rapamycin on Pten mutant mice occurred despite its opposite effect on control mice (Fig. 7B). This trend was also seen when the mice were reexposed to the same social target $3 \mathrm{~d}$ later. Thus, rapamycin appears to specifically prevent development of anxiety and social behavior abnormalities in Pten mutants when administered early.

We attempted to perform these same tests on the older group of treated mice; however, the older mutant mice were more vulnerable to handling, and increased mortality during the period of injection was observed, making the tests difficult to perform and analyze. As we suspected, chronic daily injection of rapamycin altered behavior directly as a consequence of stress placed on the mice. This effect was apparently amplified in the mutant mice that already exhibited a dramatic increase in anxiety.

Pten mutant mice also develop an age-related increase of spontaneous seizures (Ogawa et al., 2007). We therefore investigated whether rapamycin exposure would have an effect on sei- zure occurrence. As shown in Figure 7C, EEG/EMG monitoring was performed over a period of 5 weeks for each mouse. EEG/ EMG signals were recorded each week on 3 consecutive days (Tuesday to Thursday). For each mouse, baseline EEG/EMG was recorded at the first week. Then mutant mice were divided into two groups that were treated with either rapamycin or vehicle starting at the beginning of the second week. As anticipated, most mutant mice $(n=11)$ developed spontaneous seizures during the period of study, with the exception of one mouse in the drugtreated group that only displayed occasional epileptiform activity (e.g., spikes and spike waves) but not full seizures. During the seizures, repetitive spike-wave patterns were noted, sometimes accompanied by rhythmic slow activity. Continuous spike-wave bursting was also seen. In the baseline EEG/EMG recording, although the severity of the seizures varied between individual animals, there was no significant difference in either frequency or duration of the seizures between vehicle- and rapamycin-treated groups. However, compared with baseline, the mean duration of the seizures decreased significantly in the drug-treated group in 

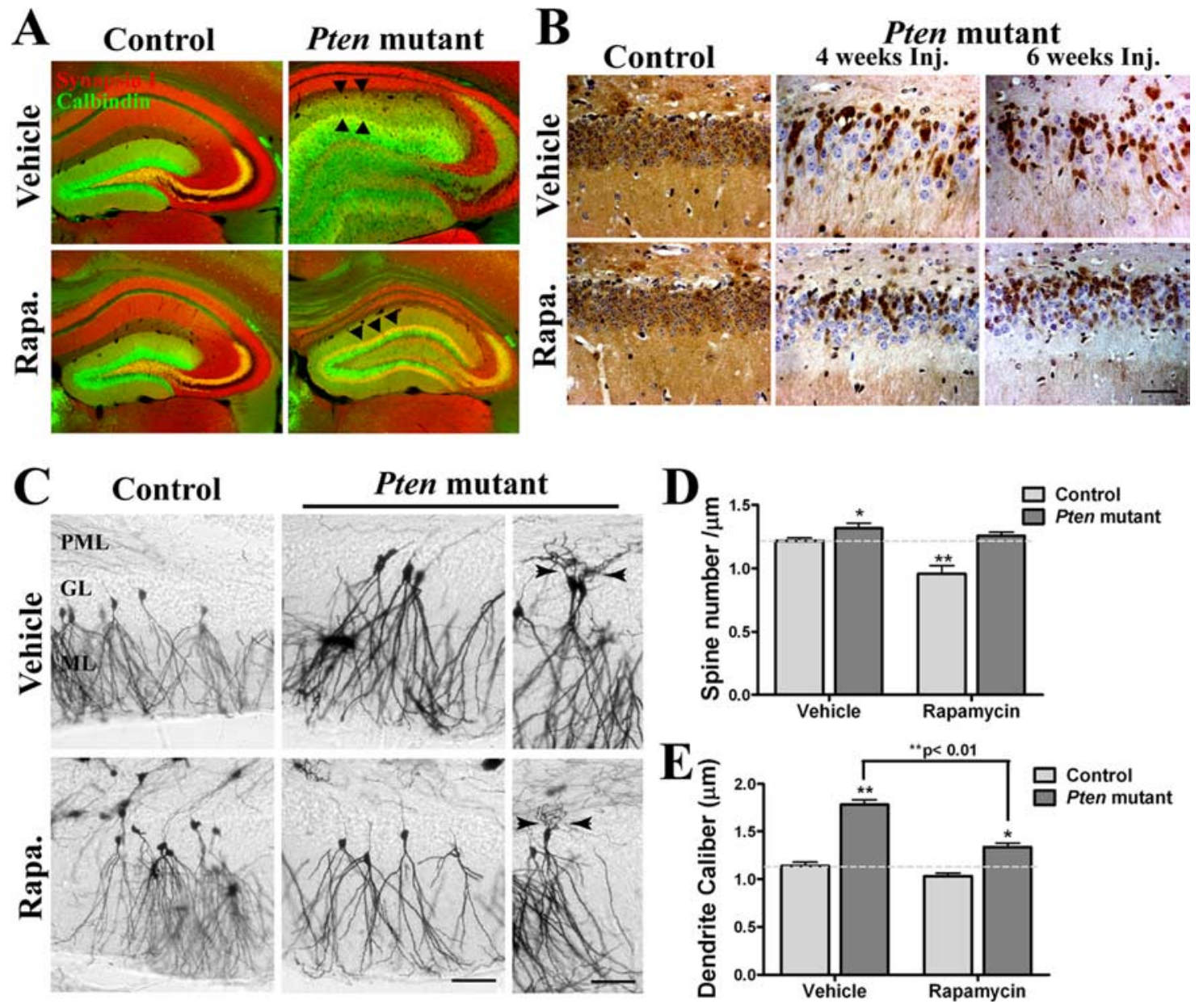

Figure 6. Rapamycin reverses dentate gyrus enlargement but not ectopic axonal projections when applied to adult Pten mutant mice. $\boldsymbol{A}$, Immunostaining shows that rapamycin (Rapa) substantially reverses dentate gyrus hypertrophy, but axonal ectopia (arrowheads) still persist after 6 weeks of treatment in adult Pten mutant mice. Scale bar, $500 \mu \mathrm{m}$. B, PTEN immunostaining of dentate granule cells demonstrates reversal of soma hypertrophy of Pten-negative cells and restored granular layer organization after rapamycin treatment. Scale bar, $50 \mu \mathrm{m}$. $C$, Representative pictures of Golgi staining show reversal of neuronal hypertrophy in the dentate gyrus of Pten mutant mice after 4 weeks of rapamycin treatment. PML, Polymorphic layer; GL, granular layer. Scale bar, $50 \mu \mathrm{m} . \boldsymbol{D}, \boldsymbol{E}$, Quantification shows that increases in spine density and dendritic caliber in Pten mutant mice were decreased after rapamycin treatment. $n=3$ brains were analyzed for each group. Five to ten primary dendritic branches were counted for each brain. Data are mean \pm SEM and were analyzed by ANOVA, followed by post hoc $t$ test. ${ }^{*} p<0.05$ and ${ }^{* *} p<0.01$ compared with control mice treated with vehicle or between groups as indicated.

week 3 and 4 (Fig. 6D) $\left(F_{(4,25)}=3.172 ; p<0.05\right)$. This decrease was not observed in the vehicle-treated group (Fig. 7D). In addition, the tendency toward reduced seizure occurrence approached significance in the drug-treated group, despite large individual variance (Fig. 7D). It is also worth mentioning that two mutant mice from the vehicle group developed severe status epilepticus as the study progressed, resulting in premature death during the third week of treatment. Wild-type control mice $(n=$ 3 ) treated with rapamycin did not develop seizures or display adverse effects. Thus, pharmacologic blockade of mTORC1 was sufficient to quantitatively reverse seizure behavior in Pten mutant mice.

\section{Feedback effects on AKT}

It has been suggested that chronic rapamycin exposure can have negative effects on AKT activity (Sarbassov et al., 2005, 2006). Studies show that mTORC2 can effectively phosphorylate AKT at serine 473, which is important for AKT activity. Although only mTORC1 is rapamycin sensitive, long-term rapamycin treatment can cause the depletion of mTOR from mTORC2, thereby negatively affecting AKT activity. However, this feedback effect is context dependent, because in vitro not every cell line exposed to rapamycin results in downregulation of P-AKT-S473 levels (Sarbassov et al., 2006). To examine potential chronic effects of rapamycin on AKT activity in our system, we collected cortical and hippocampal tissues at different postinjection time points for Western blotting analysis of P-AKT-S473 levels. The data indicate that AKT-S473 phosphorylation is reduced by chronic rapamycin exposure in both treated controls and mutants (Fig. $8 \mathrm{~A}$ ). This trend is clearly seen after the first week of drug exposure and persists in the following injection weeks. However, the overall P-AKT-S473 level in drug-treated mutant mice remained higher than that in vehicle-treated controls, as also supported by $\mathrm{P}$ AKT-S473 immunostaining of the dentate gyrus (Fig. $8 \mathrm{~B}$ ). A similar effect was observed in the cortex (data not shown). Therefore, we are unable to exclude the possibility that rapamycin, in part, exerts its effect on cellular morphology and behavior through this feedback action, thus potentially implicating additional effectors downstream of AKT.

\section{TSC1-mediated neuronal hypertrophy in vivo}

Previous in vitro studies have reported that loss of TSC1/2 or overactivation of mTOR signaling causes neuronal hypertrophy and increased dendritic arborization (Jaworski et al., 2005; Ku- 
mar et al., 2005; Tavazoie et al., 2005). In vivo, embryonic loss of Tsc1 in neurons driven by Synapsin I-cre causes severe seizures in mice, although only one of two independent studies observed obvious cortical tubers similar to those found in human TSC patients (Meikle et al., 2007; Wang et al., 2007). To further confirm the role of the mTOR pathway in cell morphology in our system, we generated Tsc1 conditional knock-out mice (Tsc1 mutant mice hereafter) using our postnatal Nsecre line. Although the progeny of this cross had reduced viability (data not shown), we were able to examine three $T s c 1$ mutants that survived to 10 weeks of age. Interestingly, although the Tsc1 mutants examined were $\sim 20 \%(20.1 \pm 9.2 \%)$ smaller than their littermate controls, they showed a $36 \%(36.5 \pm 14.8 \%)$ increase in brain/ body weight ratio. Closer examination demonstrated giant neurons in cortex (supplemental Fig. 4A, arrowhead, available at www.jneurosci.org as supplemental material), which resembled the cortical tubers observed in human TSC patients. In addition, the dentate gyrus in Tscl mutants was obviously enlarged (supplemental Fig. $4 A$, available at www.jneurosci.org as supplemental material). Additional analysis indicated significantly increased thickness of mossy fiber tract $(p<0.01)$ and molecular layers $(p<0.05)$, suggesting axonal and dendritic hypertrophy (Fig. $8 C)$. Also, P-S6 levels were increased in the cortex and hippocampus of Tsc1 mutant mice, reflecting robust activation of mTOR signaling (supplemental Fig. 4B, available at www.jneurosci.org as supplemental material). All of these changes were similar to those observed in Pten mutant mice. These independent genetic data further support the key role of the PI3K/AKT/ mTOR pathway in mediating the anatomical defects seen in our mutant mice.

\section{Discussion}

The development and use of murine models for the purpose of replicating and better understanding human disease has gained considerable ascendance in recent years. Here, we take advantage of murine models to attempt to better understand the etiology of human macrocephaly and autism when associated with the PI3K/ AKT signaling pathway.

ASDs represent a broad range of developmental brain disorders characterized by impairment of communication and social interactions and frequently accompanied by restricted and repetitive behaviors. One of the frequent physical traits associated with autism is abnormal brain size. Both macrocephaly and microcephaly have been reported in autism patients. We are particularly interested in the $10-20 \%$ of autism patients with associated macrocephaly (Piven et al., 1995; Davidovitch et al., 1996; Lainhart et al., 1997; Stevenson et al., 1997; Fidler et al., 2000; Deutsch and Joseph, 2003; Lainhart, 2003). A recent review from Courchesne et al. (2007) suggests that overgrowth during early
B

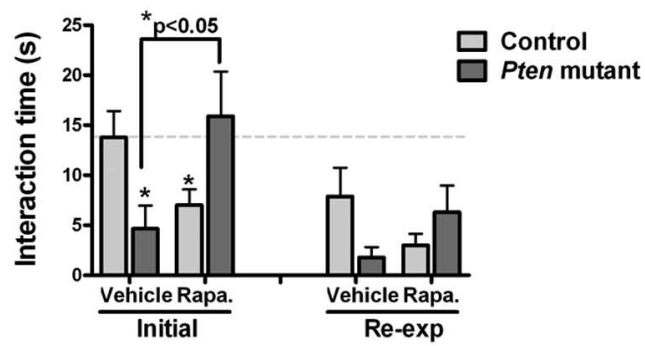

Seizure duration

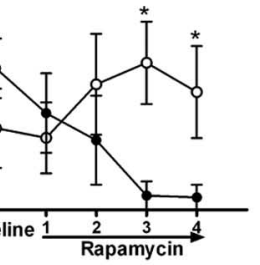

Rapa injection for 4 weeks

1st week 2nd week 3 rd week 4 th week

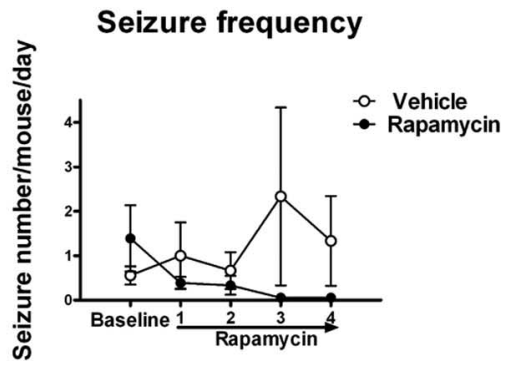

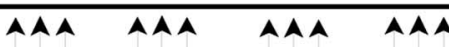

Figure 7. Rapamycin treatment reduces anxiety, improves social activity, and controls seizures. $A$, In the open-field test, group. ${ }^{*} p<0.05$ compared between vehicle- and rapamycin-treated mutants. Data are mean \pm SEM and were analyzed by ANOVA, followed by post hoc $t$ test.

brain development could be one of the key factors in the pathogenesis of autism. Thus, one hypothesis would be that pathways that control growth could play an important role in the etiology of autism. One such pathway is the PI3K/AKT pathway, which regulates cell growth and division. Mutations in PTEN, the negative regulator of this pathway, have been found to cause macrocephaly in both human and mouse. In addition, increasing clinical reports link germ-line PTEN mutations to autism patients with macrocephaly (Goffin et al., 2001; Butler et al., 2005; Herman et al., 2007a,b). Consistent with these clinical reports, we demonstrated previously that mice with Pten loss in postmitotic neurons in the cortex and hippocampus develop macrocephaly and additional behavioral phenotypes that are reminiscent of human autism (Kwon et al., 2006a). This information, coupled with the link of autism to tuberous sclerosis, indicates that dysregulation of the PI3K/AKT/mTORC1 pathway might be responsible for the pathogenesis of a subset of autism patients.

In our system, the abnormal behaviors observed in Pten mutant mice are accompanied by an overgrowth of the cortex and hippocampus and changes in neuronal morphology, including neuronal hypertrophy and loss of neuronal polarity. Blocking the TSC/mTORC1 downstream pathway in Pten mutants dramatically reversed the soma, dendritic, and axonal hypertrophy in 

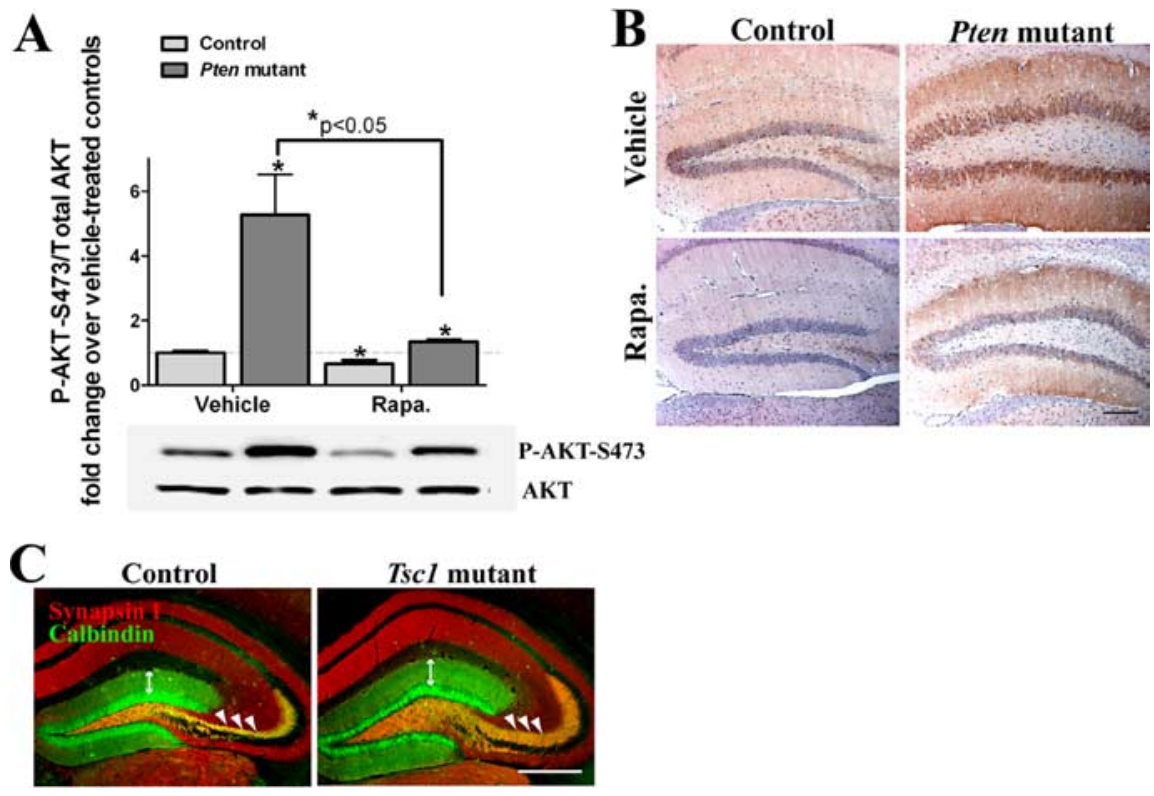

Figure 8. Long-term rapamycin treatment has a feedback effect on AKT activity and loss of Isc I causes a similar neuronal hypertrophy phenotype as Pten loss. A, Rapamycin (Rapa) treatment has a negative feedback effect on P-AKT-S473 level as shown by Western blotting on hippocampus. $n=3$ mice per group. Data are mean \pm SEM and analyzed by $t$ test. ${ }^{*} p<0.05$ compared with control mice treated with vehicle. B, P-AKT-S473 immunostaining of the dentate gyrus of adult mice treated with either vehicle or rapamycin for 4 weeks. Scale bar, $200 \mu \mathrm{m}$. C, Representative immunostaining pictures show increased thickness of mossy fiber tract (arrowheads) and molecular layer (arrows) in Tsc 1 mutant mice. Scale bar, $500 \mu \mathrm{m} . n=3$ pairs of mice were analyzed.

both young and old groups. However, the loss of polarity was not always inhibited by rapamycin treatment. When applied to asymptomatic Pten mutants, rapamycin suppressed the appearance of Synapsin I staining in the molecular layer, a sign of loss of polarity of granule cells, whereas in older mice, ectopic Synapsin I staining was reduced but not eliminated. The overall inhibitory effect of rapamycin on ectopic axonal projection is consistent with a recent study on the role of the TSC/mTORC1 pathway in controlling neuronal polarity (Choi et al., 2008). It is not likely solely attributable to the feedback effect, because the basal level of pAKT-S473 in drug-treated Pten mutants is still higher than that in vehicle-treated controls. There are a couple of possible explanations for why rapamycin treatment did not fully reverse the ectopic projection phenotype in older mice. We suspect that established polarity loss may become fixed and not subject to reversibility in vivo. Alternatively, other PI3K/AKT downstream pathways may also be at play. Thus, ectopic axonal projection may result from activation of multiple AKT downstream pathways, including the TSC/mTORC1 pathway. The fact that we did not observe ectopic Synapsin I staining in our Tscl mutants would be consistent with this latter explanation. However, we should mention that the appearance of ectopic Synapsin I staining was mainly observed in older Pten mutant mice. Unfortunately, the failure of $T s c 1$ mutant mice to thrive, leading to death of the mice before adulthood, precluded this analysis. We believe that death results from kidney failure, although this has yet to be rigorously ascertained (data not shown).

Previous studies have conditionally ablated Pten in the brain using a murine GFAP-cre transgenic line ( $m G F A P-c r e)$ (Backman et al., 2001; Kwon et al., 2001). The mutant mice developed dramatic brain abnormalities especially in the cerebellum and hippocampus, accompanied by macrocephaly, severe seizures, and increased morbidity. The broad and early expression of the cre transgene used for the studies, however, limited interpretation about the precise nature of direct versus indirect defects. Kwon et al. (2003) further used a rapamycin analog, CCI-779, to treat the $m G F A P-c r e ; P t e n^{\text {loxP/loxP }}$ mutant mice and reported partial inhibition of cell size growth in soma. This study further qualitatively described appearance of seizures (by investigator observation) in mutant mice that was reduced after drug treatment.

The present study is consistent with previous reports, but it has several important distinctions. First, in contrast to broad and early deletion of the tumor suppressors, we restricted Pten and Tscl ablation to a limited number of postnatal, postmitotic hippocampal and cortical neurons. Second, our study on neuronal morphology is not only restricted to soma hypertrophy but also extends to dendritic and axonal hypertrophy and neuronal polarity. Third, we observed similar anatomical and cellular hypertrophy in Nse-cre; $T s c 1^{\text {loxP/loxP }}$ mice, providing additional genetic support for the role of this pathway in the phenotype. Finally, we found the cellular effects of rapamycin could relate to quantifiable behavioral outcomes including reduced anxiety, increased social interest, and control of seizure duration and frequency measured by objective EEG measurements. Therefore, our mouse models permit us to examine the mutant neurons and to identify the anatomical regions responsible for the defects. With that, we are able to assert that postmitotic neurons can undergo dramatic morphological changes during deregulation of the PI3K/AKT pathway and relate these changes to abnormal behaviors.

Although our Tsc1 mutant mice failed to thrive, our conclusion regarding the role of $T s c 1$ in the observed neuronal phenotypes is supported by an increasing number of reports from other groups that suggest that deletion of Tscl in nervous system causes neuronal hypertrophy and seizures (Uhlmann et al., 2002; Meikle et al., 2007; Wang et al., 2007). As our manuscript was in preparation, studies from other laboratories reported that applying rapamycin could control seizures in human GFAP-cre; Tsc1 loxP/loxP mice (Zeng et al., 2008) and prolong the survival of Synapse I-cre; Tscl ${ }^{\text {loxP/loxP }}$ mutant mice (Meikle et al., 2008), further supporting our findings here. Twenty to twenty-five percent of autism patients experience seizures, which is life threatening (Canitano, 2007). In our study, we used EEG recording, the most objective measurement method, to monitor seizure activity. We were surprised by the degree to which rapamycin administration could reverse both the seizure frequency and duration. The anti-seizure effect of the drug was observed as early as the first week of treatment and remained throughout the 4 week drug administration period. In contrast, Pten mutants treated with vehicle demonstrated higher seizure frequency and duration, with two of six mice perishing during the injection period. These data reveal that rapamycin is a potentially useful seizure treatment modality for autism patients, particularly for those bearing germ-line Pten or Tsc1/2 mutations. More importantly, a recent study suggested that $T s c 2^{+/-}$mice show deficits in learning and memory that could be reversed by rapamycin treatment (Ehninger et al., 2008). Similarly, $T s c 1^{+/-}$mice display cognitive deficits, including impaired social behaviors (Goorden et al., 2007). These data 
indicate the possible involvement of the TSC/mTORC1 pathway in autism-related behaviors in human. In our social tests, rapamycin improved the social interaction behavior of Pten mutant mice despite an opposite effect on wild-type controls, which not only is consistent with these previous reports but also suggests the importance of this pathway in maintaining normal social activity.

We should mention that rapamycin can also modify the activity of ryanodine receptors (RyRs), a family of calcium-release channels. It is known that FKBP12 binds to members of the RyR family and that binding of rapamycin to FKBP12 releases it from RyRs, resulting in increased channel-opening probability (Chelu et al., 2004). Although to date there is no evidence suggesting that RyRs regulate neuronal morphology, they may influence synaptic activity under certain circumstances (Shimizu et al., 2008). Therefore, we cannot exclude the possibility that rapamycin functions through RyRs as well.

In conclusion, we performed the present study in the context of seeking possible mechanisms underlying PI3K pathwayassociated human autism. This approach may help to shed light on the anatomical and cellular underpinnings of related brain disorders and, furthermore, provides evidence for a potential therapeutic window in certain cases of ASD.

\section{References}

Ali IU, Schriml LM, Dean M (1999) Mutational spectra of PTEN/MMAC1 gene: a tumor suppressor with lipid phosphatase activity. J Natl Cancer Inst 91:1922-1932.

Backman SA, Stambolic V, Suzuki A, Haight J, Elia A, Pretorius J, Tsao MS, Shannon P, Bolon B, Ivy GO, Mak TW (2001) Deletion of Pten in mouse brain causes seizures, ataxia and defects in soma size resembling Lhermitte-Duclos disease. Nat Genet 29:396-403.

Butler MG, Dasouki MJ, Zhou XP, Talebizadeh Z, Brown M, Takahashi TN, Miles JH, Wang CH, Stratton R, Pilarski R, Eng C (2005) Subset of individuals with autism spectrum disorders and extreme macrocephaly associated with germline PTEN tumour suppressor gene mutations. J Med Genet 42:318-321

Canitano R (2007) Epilepsy in autism spectrum disorders. Eur Child Adolesc Psychiatry 16:61-66.

Chelu MG, Danila CI, Gilman CP, Hamilton SL (2004) Regulation of ryanodine receptors by FK506 binding proteins. Trends Cardiovasc Med 14:227-234

Chemelli RM, Willie JT, Sinton CM, Elmquist JK, Scammell T, Lee C, Richardson JA, Williams SC, Xiong Y, Kisanuki Y, Fitch TE, Nakazato M, Hammer RE, Saper CB, Yanagisawa M (1999) Narcolepsy in orexin knockout mice: molecular genetics of sleep regulation. Cell 98:437-451.

Choi YJ, Di Nardo A, Kramvis I, Meikle L, Kwiatkowski DJ, Sahin M, He X (2008) Tuberous sclerosis complex proteins control axon formation. Genes Dev 22:2485-2495.

Courchesne E, Pierce K, Schumann CM, Redcay E, Buckwalter JA, Kennedy DP, Morgan J (2007) Mapping early brain development in autism. Neuron 56:399-413.

Danscher G, Stoltenberg M, Bruhn M, Søndergaard C, Jensen D (2004) Immersion autometallography: histochemical in situ capturing of zinc ions in catalytic zinc-sulfur nanocrystals. J Histochem Cytochem 52:1619-1625.

Davidovitch M, Patterson B, Gartside P (1996) Head circumference measurements in children with autism. J Child Neurol 11:389-393.

Deutsch CK, Joseph RM (2003) Brief report: cognitive correlates of enlarged head circumference in children with autism. J Autism Dev Disord 33:209-215.

Ehninger D, Han S, Shilyansky C, Zhou Y, Li W, Kwiatkowski DJ, Ramesh V, Silva AJ (2008) Reversal of learning deficits in a Tsc2 +/ - mouse model of tuberous sclerosis. Nat Med 14:843-848.

Fidler DJ, Bailey JN, Smalley SL (2000) Macrocephaly in autism and other pervasive developmental disorders. Dev Med Child Neurol 42:737-740.

Goffin A, Hoefsloot LH, Bosgoed E, Swillen A, Fryns JP (2001) PTEN mutation in a family with Cowden syndrome and autism. Am J Med Genet 105:521-524.

Goorden SM, van Woerden GM, van der Weerd L, Cheadle JP, Elgersma Y
(2007) Cognitive deficits in Tsc1 $+/-$ mice in the absence of cerebral lesions and seizures. Ann Neurol 62:648-655.

Heitman J, Movva NR, Hall MN (1991) Targets for cell cycle arrest by the immunosuppressant rapamycin in yeast. Science 253:905-909.

Herman GE, Butter E, Enrile B, Pastore M, Prior TW, Sommer A (2007a) Increasing knowledge of PTEN germline mutations: two additional patients with autism and macrocephaly. Am J Med Genet A 143:589-593.

Herman GE, Henninger N, Ratliff-Schaub K, Pastore M, Fitzgerald S, McBride KL (2007b) Genetic testing in autism: how much is enough? Genet Med 9:268-274.

Inoki K, Li Y, Zhu T, Wu J, Guan KL (2002) TSC2 is phosphorylated and inhibited by Akt and suppresses mTOR signalling. Nat Cell Biol 4:648-657.

Jaworski J, Spangler S, Seeburg DP, Hoogenraad CC, Sheng M (2005) Control of dendritic arborization by the phosphoinositide-3'-kinase-Aktmammalian target of rapamycin pathway. J Neurosci 25:11300-11312.

Kumar V, Zhang MX, Swank MW, Kunz J, Wu GY (2005) Regulation of dendritic morphogenesis by Ras-PI3K-Akt-mTOR and Ras-MAPK signaling pathways. J Neurosci 25:11288-11299.

Kwon CH, Zhu X, Zhang J, Knoop LL, Tharp R, Smeyne RJ, Eberhart CG, Burger PC, Baker SJ (2001) Pten regulates neuronal soma size: a mouse model of Lhermitte-Duclos disease. Nat Genet 29:404-411.

Kwon CH, Zhu X, Zhang J, Baker SJ (2003) mTor is required for hypertrophy of Pten-deficient neuronal soma in vivo. Proc Natl Acad Sci U S A 100:12923-12928.

Kwon CH, Zhou J, Li Y, Kim KW, Hensley LL, Baker SJ, Parada LF (2006a) Neuron-specific enolase-cre mouse line with cre activity in specific neuronal populations. Genesis 44:130-135.

Kwon CH, Luikart BW, Powell CM, Zhou J, Matheny SA, Zhang W, Li Y, Baker SJ, Parada LF (2006b) Pten regulates neuronal arborization and social interaction in mice. Neuron 50:377-388.

Lainhart JE (2003) Increased rate of head growth during infancy in autism. JAMA 290:393-394.

Lainhart JE, Piven J, Wzorek M, Landa R, Santangelo SL, Coon H, Folstein SE (1997) Macrocephaly in children and adults with autism. J Am Acad Child Adolesc Psychiatry 36:282-290.

Luikart BW, Nef S, Virmani T, Lush ME, Liu Y, Kavalali ET, Parada LF (2005) TrkB has a cell-autonomous role in the establishment of hippocampal Schaffer collateral synapses. J Neurosci 25:3774-3786.

Manning BD, Tee AR, Logsdon MN, Blenis J, Cantley LC (2002) Identification of the tuberous sclerosis complex-2 tumor suppressor gene product tuberin as a target of the phosphoinositide 3-kinase/akt pathway. Mol Cell 10:151-162.

Marsh DJ, Kum JB, Lunetta KL, Bennett MJ, Gorlin RJ, Ahmed SF, Bodurtha J, Crowe C, Curtis MA, Dasouki M, Dunn T, Feit H, Geraghty MT, Graham JM Jr, Hodgson SV, Hunter A, Korf BR, Manchester D, Miesfeldt S, Murday VA, Nathanson KL, Parisi M, Pober B, Romano C, Eng C (1999) PTEN mutation spectrum and genotype-phenotype correlations in Bannayan-Riley-Ruvalcaba syndrome suggest a single entity with Cowden syndrome. Hum Mol Genet 8:1461-1472.

Meikle L, Talos DM, Onda H, Pollizzi K, Rotenberg A, Sahin M, Jensen FE, Kwiatkowski DJ (2007) A mouse model of tuberous sclerosis: neuronal loss of Tscl causes dysplastic and ectopic neurons, reduced myelination, seizure activity, and limited survival. J Neurosci 27:5546-5558.

Meikle L, Pollizzi K, Egnor A, Kramvis I, Lane H, Sahin M, Kwiatkowski DJ (2008) Response of a neuronal model of tuberous sclerosis to mammalian target of rapamycin (mTOR) inhibitors: effects on mTORC1 and Akt signaling lead to improved survival and function. J Neurosci 28:5422-5432.

Ogawa S, Kwon CH, Zhou J, Koovakkattu D, Parada LF, Sinton CM (2007) A seizure-prone phenotype is associated with altered free-running rhythm in Pten mutant mice. Brain Res 1168:112-123.

Piven J, Arndt S, Bailey J, Havercamp S, Andreasen NC, Palmer P (1995) An MRI study of brain size in autism. Am J Psychiatry 152:1145-1149.

Potter CJ, Pedraza LG, Xu T (2002) Akt regulates growth by directly phosphorylating Tsc2. Nat Cell Biol 4:658-665.

Sarbassov DD, Guertin DA, Ali SM, Sabatini DM (2005) Phosphorylation and regulation of $\mathrm{Akt} / \mathrm{PKB}$ by the rictor-mTOR complex. Science 307:1098-1101.

Sarbassov DD, Ali SM, Sengupta S, Sheen JH, Hsu PP, Bagley AF, Markhard AL, Sabatini DM (2006) Prolonged rapamycin treatment inhibits mTORC2 assembly and Akt/PKB. Mol Cell 22:159-168. 
Shimizu H, Fukaya M, Yamasaki M, Watanabe M, Manabe T, Kamiya H (2008) Use-dependent amplification of presynaptic $\mathrm{Ca}^{2+}$ signaling by axonal ryanodine receptors at the hippocampal mossy fiber synapse. Proc Natl Acad Sci U S A 105:11998-12003.

Smalley SL (1998) Autism and tuberous sclerosis. J Autism Dev Disord 28:407-414.

Smalley SL, Tanguay PE, Smith M, Gutierrez G (1992) Autism and tuberous sclerosis. J Autism Dev Disord 22:339-355.

Soriano P (1999) Generalized lacZ expression with the ROSA26 Cre reporter strain. Nat Genet 21:70-71.

Stevenson RE, Schroer RJ, Skinner C, Fender D, Simensen RJ (1997) Autism and macrocephaly. Lancet 349:1744-1745.

Suzuki A, Yamaguchi MT, Ohteki T, Sasaki T, Kaisho T, Kimura Y, Yoshida R, Wakeham A, Higuchi T, Fukumoto M, Tsubata T, Ohashi PS, Koyasu S, Penninger JM, Nakano T, Mak TW (2001) T cell-specific loss of Pten leads to defects in central and peripheral tolerance. Immunity 14:523534.

Tavazoie SF, Alvarez VA, Ridenour DA, Kwiatkowski DJ, Sabatini BL (2005) Regulation of neuronal morphology and function by the tumor suppressors Tsc1 and Tsc2. Nat Neurosci 8:1727-1734.
Uhlmann EJ, Wong M, Baldwin RL, Bajenaru ML, Onda H, Kwiatkowski DJ, Yamada K, Gutmann DH (2002) Astrocyte-specific TSC1 conditional knockout mice exhibit abnormal neuronal organization and seizures. Ann Neurol 52:285-296.

Ulfig N, Nickel J, Bohl J (1998) Monoclonal antibodies SMI 311 and SMI 312 as tools to investigate the maturation of nerve cells and axonal patterns in human fetal brain. Cell Tissue Res 291:433-443.

Wang Y, Greenwood JS, Calcagnotto ME, Kirsch HE, Barbaro NM, Baraban SC (2007) Neocortical hyperexcitability in a human case of tuberous sclerosis complex and mice lacking neuronal expression of TSC1. Ann Neurol 61:139-152.

Wiznitzer M (2004) Autism and tuberous sclerosis. J Child Neurol 19:675-679.

Zeng LH, Xu L, Gutmann DH, Wong M (2008) Rapamycin prevents epilepsy in a mouse model of tuberous sclerosis complex. Ann Neurol 63:444-453.

Zhou XP, Marsh DJ, Morrison CD, Chaudhury AR, Maxwell M, Reifenberger G, Eng C (2003) Germline inactivation of PTEN and dysregulation of the phosphoinositol-3-kinase/Akt pathway cause human LhermitteDuclos disease in adults. Am J Hum Genet 73:1191-1198. 